\title{
La prévention des risques liés à la sécheresse géotechnique : une expérience transposable à d'autres aléas naturels
}

\section{TOULEMONT}

Géologue, ex-Chargé de mission au MEDD, DPPR. 49 boulevard de Port-Royal 75013 Paris, m.toulemont@wanadoo.fr

\section{R. COJEAN}

Ecole des mines de Paris, Centre de Géosciences

UMLV - Bâtiment IFI

5 boulevard Descartes. Champs-sur-Marne 77454 Marne-la-Vallée Cedex roger.cojean@ensmp.fr
Cette note présente un point de vue sur l'expèrience acquise dans l'analyse et la cartographie de l'aléa sécheresse géotechnique, la mise en oeuvre de polítiques de prévention des risques associés, l'information apportée aux différents acteurs de l'acte de construire ainsi qu'aux particuliers sur le sujet du retrait/gonflement des sols argileux. Elle se conclut par une ouverture vers des préoccupakions de prévention relatives à d'autres aléas naturels, considérés dans la perspective du changement climatique global.

Mots-clés : aléa sécheresse géotechnique, retrait gonflement, sols argileux, cartographie, prévention, information, changement climatique global.

\section{Risk prevention related to shrink/ swell hazard: an experience useful for other natural hazards}

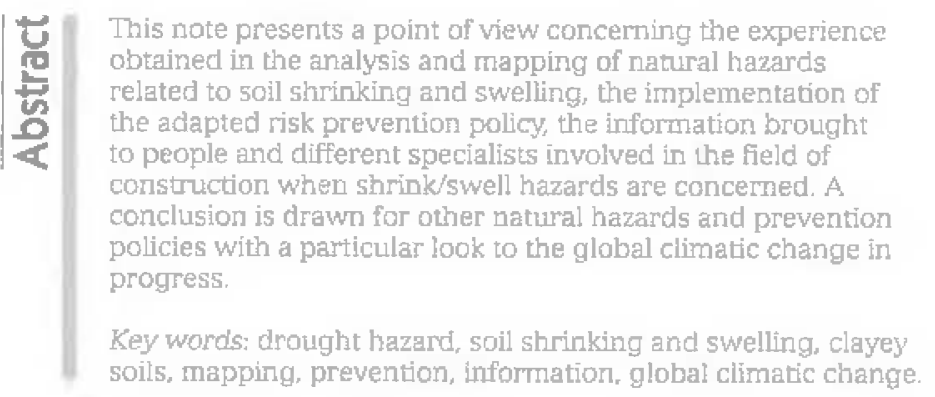




\section{Introduction}

La Journée technique organisée le 18/01/2007. conjointement par le Comité français de géologie de l'ingénteur et de l'environnement (CFGI) et le Comité français de mécanique des sols et de géotechnique (CFMS), sur le thème de la sécheresse géotechnique a montré l'actualité des préoccupations scientifiques et techniques relatives à ce sujet, pour des domaines relevant de la géologie, de I'engineering geology et de la géotechnique, de la construction sur sols argileux et de la prévention des risques. Ĺimportance des dommages dus aux phénomènes de retrait/gonflement des sols argíleux depuis 1989 et la quasi-certitude de leur renouvellement en cas de nouveatux épisodes de sécheresse intense et durable, ont conduit à la mise en cuvre d'une politique de prévention destinée à réclutre le niveau de sinistralité. Cette politique de prévention est aujourd'hui encore en cours d'adaptation dans un contexte naturel et technicue d"une grande complexité. Cet article est un point de vue que les auteurs onl tenté de placer dans le cadre du changement climatique global.

\section{2}

\section{Un bref historique de la démarche de prévention et d'information concernant la sécheresse géotechnique}

Les processus de retrait/gonflement des sols argtleux font lobjet de travaux de recherche scientifique depuis de longues années : caractérisation des facteurs de prédisposition, identification des facteurs déclenchants, recherche d'indicateurs de sécheresse géotechnique pertinents. Les refours d'expêriences et rêsuitats d'expertises techniques sur le sujet de la sécheresse géotechnique apportent leur contribution à ces recherches. On ne citera, à titre d'exemple que quelques références sur ce sujet : Philipponnat (1991), Vandangeon (1992), Al-Rawas et al. (2006).

Les principales périodes de sécheresse ayant entraîné des dommages au bâti clairement identifiés sont connues : période 1989-1992, période 1996-1997, année 2003. De 1989 ả 2002, le coût global d'indemnisation (dans le cadre du régime d'indemnisation Catastrophes naturelles ou Cat-Nat) a étê de 3,3 milliards d'euros, selon la CCR (Caisse centrale de réassurance), en deuxième poste de dépenses après les indemnisations concernant le risque inondation. La profession s'est très tôt intéressée à ce sujet technique avec différentes actions entreprises sous l'égide des ministères de l'environnement ou de l'écologie.

Ainsi, i] faut rappeler que J'Agence Qualité Construction (AQC) a faít réaliser en 1990, par un réseau d'experts, une enquête statistique sur 356 bâtiments sinistrés, afin de préciser les causes de pathologie des maisons individuetles. En 1991, le CEBTP (Centre expérimental de recherches et d'études du bâtiment et des travaux publics) a réalisé une enquête sur 412 bâtiments endommagés par la sécheresse géo016 technique. Ces études ont permis d'identítier le pro- individuelle en rez-de-chaussée $(72 \%)$, avec dallage sur terre plein $(68 \%)$, fondée entre $0,40 \mathrm{~m}$ et $0,80 \mathrm{~m}$ de profondeur $(77 \%)$, sur un sol argileux $(97 \%)$ et avec des arbres à proximité (30\%). Il en a résulté, sous l'égide des organismes: AQC, APSAD, AFAC, CCR et FNB, l'édition d'un guide pratique sur a La détermination des solutions adaptées à la réparation des désordres des bâtiments provoqués par la sécheresse » (CEBTP, 1991). La Revue française de géotechnique a publié un article consacré au retraiv/gonflement des argiles dans le $\mathbb{N}^{\circ} 57$ (octobre 1991) puis un ensemble de cinq articles dans le $n^{\circ} 58$ (janvier 1992) passant en revue les aspects scientifiques et techniques du problème et présentant les conditions d'application de la loi du 13 juillet 1982 sur l'indemnisation des victimes des catastrophes naturelles

En 1993, la Direction de la prévention des pollutions et des risques (DPPR) du ministère de l'Environnement a publié, à la Documentation française, un guide de prévention Sécheresse et construction (DPPR, 1993) présentant des recommandations à l'usage des constructeurs et énonçant les principes d'une cartographie de l'aléa et du risque sécheresse géotechnique. Ce guide, tiré à 5000 exemplaires, a été transmis en 1998 aux préfets des départements concemés et à l'ensemble des maires des communes reconnues sinistrées par arrêté inteministériel portant constatation de l'état de catastrophe naturelle. En 1993, la DPPR a élabli un document présentant la méthodologie d'une cartographie de l'aléa " sensibilité des sols à la sếcheresse " et la carte correspondante au 1/1000 000. Quatre classes d’aléa étaient distinguées, prenant en compte les caractéristiques lithologiques et géotechniques des formations géologiques ainsi que leur degré d'homogénéité et prenant appui sur l'analyse en retour des conséquences des événements "sécheresse géotechnique w disponibles à cette époque, au vu des dossiers des communes reconnues sinistrées par arrêté interministériei dans le cadre du régime d'indemnisation Cat-Nat. Les auteurs de cet article, à l'origine de cette cartographie, ont ensuite publié un article synthétique dans la revue Mappemonde (Toulemont et al, 1994).

En 1995, dans le cadre de la procédure spécifique de prise en compte des risques dans l'aménagement du territoire, au plan règlementaîre, la DPPR du ministère de l'Écologle et du Développement durable (MEDD) a lancé la réalisation des Plans de prévention des risques naturels (PPRN). A la suite des sècheresses pluriannuelles de 1989-1992, de 1996-1997, puis de la canicule de 2003, le MEDD a mandaté le BRGM afin d'établir la cartographie départementale de l'aléa retrait/gonflement au 1/50 000 sur la France entière (Vincent, 2003).

Les assureurs ont aussi progressivement apporté leur contribution à celte démarche d'information (MAIF, 2000, FFSA et GEMA, 2001).

Le Comite français de géologie de l'ingénieur et de l'environnement (CFGI) a réalisé plusieurs séances techniques relatives au thème des sols sensibles à la sécheresse : en janvier 1991, sur le thème "Sécheresse, terrains argileux et fondations $»$, puis, en janvier 2003 , sur le thème a Sols sensibles à l'eau, retours d'expérience 2 . En janvier 2007, une journée technique CFGICFMS était organisée sur le thème a Sécheresse géotechnique , accueillant plus de 150 participants.

En Grande-Bretagne, où la sécheresse de 1976 a occasionné des sinistres importants, une profondeur minimale de fondation d'1 mètre a été immédiatement rendue obligatoire pour les maisons individuelles. En 
France, malgré son intensité, cette sécheresse n'avait occasionné que peu de dommages du fait de sa brièveté, quatre mois environ, et de la faible profondeur de l'assèchement.

Il n'y a pas eu, par la suite, de modification des textes normatifs DTU $13-12$ relatifs a la profondeur hors gel $(0,50 \mathrm{~m}$ en zone tempérée) en dépit de la sécheresse géotechnique qui, depuis 1989, a constitué le premier facteur de pathologie des maisons individuelles. Cependant, l'établissement des PPR Sécheresse géotechnique et la définition d"un règlement adapté à ce type d'aléa reste aujourd'hui encore un sujet de concertation entre spécialistes.

L'importance des dégăts immobilìers durant les dernières décennies et les risques de nouvelles périodes de sécheresse ont conduit à prendre en compte la nécessité d'une politique d'information volontariste en direction des collectivités territoriales, compte teru de Ieur responsabilité en matière de prévention.

Depuis la loi du 30 julllet 2003 relative à la prévention des risques technologiques et naturels, les maires doivent en effet informer périodiquement, tous les deux ans, la population datis le cas de l'existence d'un PPR, pour une meilleure prise en compte des mesures de prévention. En situation d'attente de réalisation de PPR, il a été jugé important de signaler à l'ensemble des maîtres d'ouvrage, constructeurs de pavillons, professionnels du bâtiment, les dispositions de prévention et de protection des constructions par une information systématique et des actions de communication adaptées. C'est pourguoi le MEDD a décidé en 2004 de conduire des actions concertées avec l'Agence Qualité Construction (AQC) dont la mission essentielle est de réduire la sinistralité de $30 \%$ dans les années à venir et d'améliorer la qualité des constructions. L'AQC informe, sensibilise et motive l'ensemble des professionnels engagés dans l'acte de construire en leur faisant connaître les dispositions techniques d'ordre préventif spécifiques aw phénomène de retrait/gonflement des sols argileux. L'AQC a produit à cet effet:

- une plaquette d'information sur la prévention de ces désordres qui s'adresse à l'ensemble des lotisseurs, maittres d'ceuvre, constructeurs, contröleurs techniques et géotechniciens :

- un diaporama " sécheresse et constructions sur sol argileux : nouvelles règles préventives $\%$.

Un programme de plus de 10 rencontres interprofessionnelles régionales destinées aux professionnels et notamment aux constructeurs de maisons individuelies a été organisé et va être poursuivi. Les participants, de 100 à 200 par réunion, ont exprimé leur grande satisfaction à l'issue de ces réunions, ayant tout particulièrement apprécié le débat sur les dispositions constructives locales.

\section{3}

\section{Des sols sensibles au retrait-gonfle- ment sous toutes latitudes}

Les sols sensibles au retrajt/gonflement ou sols gonflants (expansive soilsj sont conrus pratiquement dans toutes les régions du monde. Ies dommages aux construction étant souvent très spectaculaires dans les régions arides et semi-arides. Les phylosilicates de la famille des smectites ou d'autres minéraux argileux de la famille des interstratifiés (illite-smectite...) sont toujours présents dans ces sols et leur confèrent un fort potentiel de gonflement et de retrait. Dans le cas des pays de climats tropicaux secs (régions désertiques ou semi-désertiques) et méditerranéens, où l'état de référence des sols est gënéralement un état de sol sec la plus grande partie de l'année, la sollicitation hydri. que à l’origine des dommages est représentée ie plus souvent par un apport d'eau inhabituel, générateur de processus de gonflement des sols argileux avec les dommages au bâti qui en résultent. Dans le cas des pays de climats tempérés océaniques ou continentaux, où l'état de référence des sols est un état de sol humide, la sollicitation hydrique à l'origine des dommages est le plus souvent un assèchement des sols en surface, sollicitation d'origine climatique ou liée à l'action de la végétation, génératrice de processus de retrait des sols argileux avec les dommages au bâti qui en résultent. Dans ces situations, même lorsque les minéraux argileux gonflants ne sont pas prépondêrants dans les sols concernés, comme pour certains sols argileux en Grande-Bretagne ou en France par exemple, les dommages au bâti liés à la sécheresse peuvent être très importants.

On connaît des sols sensibles au retrait/gonflement en Afrique du Sud, en Allemagne, en Argentine, en Australie, en Birmanie, au Canada, en Chine, à Chypre, à Cuba, en Espagne, en Ethiopie, en France, au Ghana, en Grande-Bretagne, en Grèce, en Inde, en Iran, en Israël, au Japon, en Jordanie, au Maroc, au Mexique, en Norvège, en Oman, en Rhodésie, en Roumarie, en Russie, en Suède, en Turquie, aux USA, au Venezuela. Certains sols gonflants sont connus sous le nom de black cotton soils aux USA et en Inde. Les géologues et géotechniciens sont familiers de ces types de sols, de même que les pédologues qui décrivent les vertisols, sols argileux riches en argiles gonflantes, soumis à un brassage par les "mouvements vertiques w liés aux variations saisonnières de volume des argiles, conduisant en périade sèche à la formation de fentes de retrait, en période humide à la formation, en surface, de micro-reliefs dénommés gilgail et au seín du matériau à la formation de surfaces de friction ou a slickensides » caractéristiques (P. Duchaufour, 1977 et 1984, D. Tessier, 2006).

A titre d'exemple, la Chine est l'un des pays du globe oủ les sols gonflants sont très largement répandus. Ils sont présents dans 20 provinces et régions occupant environ $6000000 \mathrm{~km}^{2}$. Le retrait-gonflement des sols argileux représente le principal phénomène naturel responsable des problèmes de construction sur fondations peu profondes ef de certains glissements de terrain. Selon des estimations incomplètes, le nombre de mètres carrés inhabitables pour cause de destruction suite à un gonflement du sol serait de 10 millions de mètres carrés, soit une perte économique pour la Chine estimée à environ 1 milliard de dollars par an, d'après B. Shi et al. (2002). Dans des pays comme les Etats-Unis, le Canada, le Mexicue, la Jordanie et, dans une moindre mesure, le Maghreb, les sols gonflants sont aussi très répandus. La furle prẻsence des sols gonflants constitue la cause essentielle des dégâts immobiliers, par les processus de retrait/gonflement, entraînant des coûts considérables ( 2 à 7 milliarcs de dollars aux États-Unis certaines années). 


\section{Retours d'expérience actuels transposables dans le cadre du changement climatique global}

Compte tenu de la quasj-certitude, selon le GIEC (Groupement d'experts intergouvememental sur l'étude du climat), du réchauffement climatique et de ses répercussions sur les phénomènes de sécheresse, il apparaît nécessatre, par mesure de précaution, d’accélérer les mesures de prévention et de protection actuellement en cours, afin de diminuer, à l'avenir, les effets de la sécheresse (Denhez, 2005; Durand, 2007 ; Pour la Science, 2007; Sciences et Avenirs, 2007).

D'une façon générale, d’après ce rapport, la concentration de gaz à effet de serre continue d'augmenter dans l'atmosphère, principalement du fait des activités humaines (IPCC-GIEC, 2007). L'impact sur le bilan radiatif de la Terre est important. Le climat change, avec pour la période 1995-2006 : onze des douze années les plus chaudes depuis 1850 et une augmentation de la température moyenne à la surface du globe de $0,76^{\circ} \mathrm{C}$ depuis 1860 , dont $0,74^{\circ} \mathrm{C}$ depuis 1900 et $0,20^{\circ} \mathrm{C}$ depuis 1990. D'ici 2100, suivant les différents scénarios possibles quant aux émissions de gaz à effet de serre, selon les prévisions des modèles et hors événement naturel exceptionnel, la température moyenne de l'air à la surface du globe devrait augmenter de 1,1 à $6.4^{\circ} \mathrm{C} p a 2^{\circ}$ rapport à 1990 . De plus, des changements significatifs pourraient apparaître quant aux caractéristiques de variabilité des climats, s'agissant de la fréquence et de l'intensité de certains événements climatiques extrêmes : précipitations et sécheresse (ONERC, 2003 ; E. Bard, 2006; [PCC-GIEC. 2007).

On peut donc craindre, sous les latitudes des pays tempérés, que des processus actuellement connus, mais se présentant avec de faibles intensités et des effets limités, viennent à se développer dans le futur avec de plus fortes intensités et des effets autrement dommageables, analogues à ce qui est observé aujourd'hui sous de plus basses latitudes.

Ce rapport signale aussi que le niveat des océans augmente, principalement par dilatation themicue des océans et fonte des glaces des inlandsis et des glaciers de montagne. Du fait de la grande inertie thermique des océans et de la lenteur de la fonte des glaces, cette montée des eaux se réalisera continûment pendant des siècles, même si le rếchauffement était aujourd'hui maîtrisé. Le niveau des mers a monté en moyenne de $1,8 \mathrm{~mm} / \mathrm{an}$ entre 1961 et 2003 et de $3,1 \mathrm{~mm} / \mathrm{an}$ de 1993 à 2003. L'augmentation totale durant le $x^{\prime \prime}$ siécle est estimée â $0.17 \mathrm{~m}$ (A. Cazenave, 2006).
Mais d'autres risques naturels peuvent être générés et accentués par les canicules estivales (type 2003) et les fortes précipitations hivernales, et ils ne sont pas encore vraiment pris en compte :

- recul du littoral principalement par submersion des côtes basses, localement par érosion des falaises, crayeuses ou argileuses, du fait de l'élévation accélérée du niveau de la mer. Des îles basses et des atolls sont voués à disparaître. Des régions de deltas seront submergées, les biseaux salés pénétreront plus avant dans l'arrięre pays et contamineront les réserves en eaux douces. Les zones littorales basses constituées de marais maritimes se transformeront, les pratiques aquacoles et agricoles devront évoluer;

- accroissement des incendies de forêt en période estivale et inondations en période hivernale;

- baisse des nappes phréatiques superficielles et diminution des ressources en eau:

- dépérissement agricole et forestier, eutrophisation et pollution des eaux;

- augmentation des glissements de terrain et éboulements en rapport avec les précipitations.

En s'appuyant sur l'expérience de la sécheresse géotechnique, la même démarche générale et des mesures de prévention adaptées à chaque configuration d'aléa devraient être mises en ceuvre pour ces autres risques liés au réchauffement du climat, sans attendre par mesure de précaution.

\section{5}

\section{Conclusion}

L'expérience acquise dans l’analyse de l'aléa sécheresse géotechnique et de ses effets sur les constructions mérite d'être confrontếe aux perspectives du changement climatique global. П faut espérer gue, devant les changements à venir, les écosystèmes présenteront la résilience nécessaire pour s'adapter. Les communautés administratives et sociologiques, confrontées à un milieu naturel de plus en plus stressant, devront définir dans l'urgence les bases d'un développement soutenable. La mise en ceuve des politiques de prévention des risques, incluant l'information et la formation des citoyens, doit participer de cette réaction positive.

\section{RFMERCIFWENTS}

Cette note a été établie en prenant apui su le retour d"experience acoús par lun des auteurs M. Iowemont au sein du MEDD gui est remercié. De mene IAQC est remerciée powr les informations ransmises. 
Al-Rawas A.A., Goosen M.F.A. (eds) Expansive soils. Recent advances in characterization and treatraent, 2006, $526 \mathrm{p}$.

AQC (Agence Qualite Construction) - Fordations de maisons individuelles. Principales pathologies. CD-Rom pédagogique à partir d’études de cas concrets. Diaporama retrait gonflement des sols argileux et brochure d'information. 2004

Bard E. - L'Homme face au climat. Collège de France, Odlle Jacob, 2006, 446p. Paris.

Cazenave A - Les variations actuelles du niveau de la mer : observations el causes. in Bard E., 2006, p. 85-101.

Durand F. Le réchauffernent climatique en débats. Ellipse, 2007, $188 \mathrm{p}$.

FFSA et GEMA. - Fascicule sécheresse, $2001,24 \mathrm{p}$.

CEBTP 1991. Guide pratique : Determination des solutions acaptées ã la réparation des désordres des bâtiments provoqués par la sécheresse. Sous l'égide de AOC, APSAD, AFAC, CCR, FNB - Fascicule 1: Bases de référence. 48 p., Glos. saire. Fasscicule 2: Principes et méthodologie : 8 p., Tableaux guides, Tableaux des solutions élếmentaires, Schémas de désordres et de principe de réparation, Glossaire, Fascicule 3 ; Annexes techniques, 124 p. Edition SEDITTA, París.
Derhez F. - Atlas de la menace clinatique. Autrement, coll. « Atlas/Monde n, 2005. $80 \mathrm{p}$.

DPPR - Sécheresse et construction. Guide de prévention. La Docurnentation Française, 1993, $52 \mathrm{p}$.

Duchaufour P. - Pédologie, 1 Pédogénẻse et classification. Masson, $1977,478 \mathrm{p}$.

Duchaufour P. - Pédologie. Abrégés. Mas. son, 1984,220 p.

IPCC-GIEC - Climate Change 2007: The Physical Science Basis. Summary for Polycymakers, $2007,18 \mathrm{p}$.

MAIF - La méthodologie de l’expertise us sécheresse w, $2000,19 \mathrm{p}$. et annexes.

ONERC (Observatoire national sur les effets du réchauffement climaticue en France et dans les Dom-Tom) - Conséquences du rẹchauffement climatique sur les risques liés aux êvénements météorologiques extrêmes. Actes du Collogue du 22-23 juin 2003, ONERC. Parfs, octobre 2003.

Philipponnat G. - Retrai/gonflement des argiles, proposition de méthodologie. Rev. française de géotechnique, $n^{\circ} 57$, 1991, p. 5-22.

Pour la Science, Dossier Climet, comment éviter la surchauffe, janvier/mars 2007. $120 \mathrm{p}$.

Revue française de géotechnique $\mathrm{n}^{2} 57$. octobre 1991
Revie francaise de géotechnique $n^{\circ} 58$, janvier 1992.

Sciences et Avenir, Le réchauffement climatique, $200782 \mathrm{p}$.

Shi B., Jiang H., Liu Z., Fang H.Y. Engineering geological characteristics of expansive soils in China. Engineering Geology 57, 2002, p. 63-71.

Tessier D. - Analyse comparative des politiques de prévention du risque de a sécheresse géotechnique ».Modélisation et visualisation du phénomène. Repport INRA,2006, $288 \mathrm{p}$.

Toulement M., Cojean R., Faccendini J.-P. - Cartographie prévisionnelle des sols sensibles à la sécheresse : un outil d'information prếventive. Mappemonde 4.1994, . . 2-5.

Vandangeon P. - Exemples de sinistres en région parisienne. Revue foraçaise de géotechnique, $\pi^{\circ} 58,1992$, p. 7-14.

Vincent M. 2003. Retratt/gonflement des sols argileux : méthode cartographique d'évaluation de l'aléa en vue de l'établissement de PPR. $3^{t}$ Conférence SIRNAT, Forum des Joumées pour fa prêvention des risques naturels, Orléans. Actes du Collogue, 2003, 7 p. 